\title{
Interactive comment on "The Mineral Aerosol Profiling from Infrared Radiances (MAPIR) algorithm: version 4.1 description and validation" by Sieglinde Callewaert et al.
}

\section{Anonymous Referee \#3}

Received and published: 2 April 2019

The manuscript "The Mineral Aerosol Profiling from Infrared Radiances (MAPIR) algorithm: version 4.1 description and validation" by $S$. Callewaert et al. describes a new method applied to an algorithm that retrieves mineral aerosol profiles from infrared radiances. The new version 4.1 of MAPIR and validation results are described. As stated by the authors and proved with the validation/comparison with other products, the results show a significant increase in retrieval quality.

I have several comments that need to be addressed before this paper can be accepted for publication to AMT, most of them are for the validation/comparison part.

Main comments 
I suggest combining the description of the lidars from Secs. 5.3.1-5.3.3 with the IASI description in Sect.2.

The algorithm is explained in detail, but I am a bit confused with the cloud screening. In Sect. 3.5 the IASI operational L2 cloud product is mentioned, while on P9 the failure of the EUMETSAT cloud filter is discussed as a possible reason for the cloud contamination. Is EUMETSAT cloud filter is used as an additional check?

Interactive comment

Since AERONET AOD is not measuring the IR AOD (as you discuss in Sect 5.1), I would rather call the exercise as "evaluation" rather than "validation" and suggest to change the title accordingly.

AERONET coarse AOD product includes other than dust species. According to Satheesh et al (https://doi.org/10.1029/2005GL024856), at high wind speeds, seaâĂŘsalt contributes $81 \%$ to the coarse mode. To classify (to some extent) the aerosol type, I suggest looking at the SSA product from the AERONET. The other useful reference for the discussion might be Khatri et al. (https://agupubs.onlinelibrary.wiley.com/doi/pdf/10.1002/2013JD019961).

Since the performance of the retrieval algorithm often depends on the aerosol load/type and surface contribution to the TOA reflectance, the evaluation results are not expected to be similar for e.g., low or high AOD loading conditions. Moreover, the number of cases with $A O D>0.5$ is much lower (may be up to several magnitudes lower) than the number of $A O D<0.5$ cases. Thus, the linear regression is not always the correct tool to evaluate the algorithm performance and I suggest to remove the regression line from Fig.6. Instead, I suggest looking at the AOD bias for certain AOD bins (e.g., Figs.1-2 in https://www.ncbi.nlm.nih.gov/pmc/articles/PMC6101972/ or Fig.3 in https://www.atmos-chem-phys.net/18/11389/2018/).

I have some doubts on how meaningful the mean bias of -0.04 is for the estimation of the MAPID AOD quality. To my rough estimation from Fig.7, only ca $10 \%$ of matches hit the bin which includes the number of -0.04 ; the highest probability $(\sim 0.16)$ is for

Printer-friendly version

Discussion paper 
slightly positive bias and the probability of the abs difference of $>0.1$ is high (ca $>0.40$ ). Thus, the spread is essential, even though the mean bias is low. The intercept (Table A1) is positive for most of the stations and on average is as high as ca 0.08 (my rough estimates).

The deviation of the cumulative extinction is smaller over ocean (Table1). Is that because P7L6: "we believe existing databases of ocean Ts are more reliable than land Ts" or other reasons exist? However, as for the comparison with AERONET, the results for inland stations look better than for coastal/island stations.

The transition in land/ocean AOD is not smooth (Fig 3a,b; Fig.4). Please, discuss the reason(s).

\section{Specific comments}

P2L25: OMI retrieves AOD at UV and interpolates AOD to 500nm using the Angström.

P3L15: I suggest the following changes: .... updated algorithm is presented; AOD product is evaluated against AERONET and compared.... . The work is organized as follows.

P13L10: Please, specify the wavelengths here.

Fig.6, second column. Here I suggest to color the dots with AERONET SSA, when retrieved and add the corresponding discussion on the sea salt contribution to the AERONET coarse AOD.

Fig.7 Please, add the grid.

P16L32 One period, 1-12 July 2010, is mentioned twice

Table 1. I suggest making the case specs (e.g., CALIOP, All data) more visible by adding additional horizontal line below the case name, or/and changing the fonts and/or moving the name to the left. 
P22L6: I suggest to specify months here

P23L3: Please, provide a reference, where the radar limit for the close to surface AMTD measurements is specified and discussed.

Interactive comment on Atmos. Meas. Tech. Discuss., doi:10.5194/amt-2019-84, 2019.

Interactive comment 